\title{
Nuevas tecnologías y accesibilidad académica para estudiantes con discapacidad visual
}

\section{New technologies and academic access for students with visual disabilities}

Dda. María Inés Loyola

Universidad Nacional de Córdoba

(Argentina)

loyolamariaines@yahoo.com.ar

Dra. Mónica Viada

Universidad Nacional de Córdoba

(Argentina)

monviada@yahoo.com.ar

Recibido: 15 de Diciembre de 2010

Aceptado: 20 de Febrero de 2011

\section{Resumen}

Este artículo da cuenta de las estrategias de intervención que se llevan a cabo en la Escuela de Ciencias de la Información de la UNC en el marco de la Red MATE (Materiales y Apoyos Tiflo-Educativos) para abordar la cuestión de la accesibilidad a la educación superior de estudiantes con discapacidad visual a partir del uso de las nuevas tecnologías de la información y la comunicación.

La iniciativa contempla la producción de materiales de estudio en soportes accesibles, la habilitación de un espacio público de acceso a las tiflo-TIC, la capacitación en el uso de las tecnologías y el empoderamiento del colectivo destinatario.

Entre otros logros, en dos años de trabajo se produjeron materiales digitalizados para 108 asignaturas de 12 carreras terciarias y universitarias de grado y posgrado, además de para el nivel medio de adultos. Los beneficiarios directos fueron 31, pero los materiales están disponibles para 4000 usuarios y 140 instituciones de distintos países.

Se considera que esta problemática debe ser abordada desde un enfoque multidisciplinario, que incluya aspectos relacionados con las tecnologías, pero también - y fundamentalmente- 
con el concepto de accesibilidad académica, en tanto cualidad y requisito básico que debe cumplir todo sistema educativo para permitir la inclusión igualitaria de todos y todas.

\section{Abstract}

This article explains the intervention's strategies that the Escuela de Ciencias de la Información of National University of Córdoba uses in the context of the Red MATE to aboard the accessibility to superior education for visual disabled students trough the utilization of the new technologies of information and communication.

The initiative contemplates de production of studying material in accessible supports and the enable of a public space that allows access to the tilfo-TIC. We weigh the training on the uses of technologies and the empowerment of all the addressees.

Along other achievements, in two years of work we have managed to produce digitalised materials that can be used in a 108 subjects from 12 tertiary and university careers. The direct beneficiaries where 31 but the materials are available for 400 users and 140 institutes form different countries.

It is considerate that this problematic must be faced from a multidisciplinary approach that includes matters related to technologies but also and especially to the concept of academy accessibility, being this the most important quality that any educational system must fulfil in order to allow equal inclusion for everybody.

Palabras Clave: tecnología, educación

Key Words: technology, education

Sumario: 1. Introducción. 2. Antecedentes. 3. Metodología. 4. Aplicación. 4.1. Situación contextual. 4.2. Actividades. 5. Conclusiones. 6. Bibliografía

Contents: 1. Introduction. 2. Precedents. 3. Methodology. 4. Application. 4.1. Contextual situation. 4.2. Activities. 5. Conclusion 6. Bibliography

\section{Introducción}

Este artículo da cuenta de las estrategias de intervención que se llevan a cabo en la Escuela de Ciencias de la Información de la Universidad Nacional de Córdoba, en el marco de la Red MATE, para abordar la cuestión de la accesibilidad a la educación superior de estudiantes 
con discapacidad visual, a partir del uso de las nuevas tecnologías de la información y la comunicación.

El alto nivel de deserción registrado tanto en la educación media como superior por parte de los jóvenes con discapacidad visual es la razón que motivó la elaboración de este proyecto, que se propone utilizar las nuevas tecnologías como medio para resolver las dificultades en el acceso a la educación, y favorecer así la inclusión académica de este colectivo.

De acuerdo al diagnóstico realizado con la participación de diversos actores involucrados, algunas de las razones del elevado índice de deserción escolar (en todos los niveles) de las personas con discapacidad visual son la carencia de materiales de lectura accesibles y de formación específica para el aprovechamiento de lo que hoy aportan las Tiflo-TIC, término que conjuga la tiflotecnia (tecnología aplicada a las necesidades de las personas con discapacidad visual) y la aplicación de ésta específicamente en el marco de las Tecnologías de la Información y la Comunicación (TIC). Otra causa es la falta de información dirigida a docentes y profesionales respecto de las posibilidades que brindan las herramientas informáticas a los/as estudiantes con discapacidad visual insertos en cualquiera de los niveles educativos.

La iniciativa surgió teniendo como eje fundamental el trabajo en red, la suma de experiencias y la articulación entre las organizaciones de la sociedad civil que trabajan en la temática, las universidades y otros centros educativos.

De esta forma se conformó la Red MATE, una iniciativa conjunta de la Escuela de Ciencias de la Información de la Universidad Nacional de Córdoba, la Asociación Civil Tiflonexos (Argentina) y la Unión Nacional de Ciegos del Uruguay y tiene como objetivo incrementar los niveles educativos alcanzados por los y las jóvenes con discapacidad visual a través del uso eficiente de las tiflo-TIC.

En síntesis, el proyecto, entonces, se propone promover la autonomía y la igualdad de oportunidades en el acceso a la educación superior para estudiantes con discapacidad visual en las áreas de ciencias sociales y humanas a través del uso de las tiflo-TIC.

El trabajo se organizó en torno a cuatro ejes:

- Producción de textos en soportes accesibles

- Acceso público a las Tiflo-TIC

- Capacitación en el uso de las Tiflo-TIC

- Empoderamiento. 


\section{Antecedentes}

Si bien en la Argentina no se cuenta con datos precisos, se sabe que el acceso a la educación superior para una persona con discapacidad visual resulta más complicado que para una persona sin discapacidad. Esto hace que, al no contar con los apoyos necesarios, sea bajo el porcentaje de personas con discapacidad visual que acceden a la universidad o que su rendimiento no sea el adecuado y, por ende, la deserción sea mayor.

Según la Encuesta Nacional de Discapacidad, realizada por el INDEC (2002- 2003), existían, en 2003, 17.000 personas con discapacidad estudiando en el nivel superior, lo que representa un $1,2 \%$ del total de los estudiantes en general. Esto indica una desventaja para el acceso, pues en esta misma encuesta, se calculó que en la franja de edades entre 20 y 30 años existe un $5 \%$ de personas con discapacidad.

El citado documento revela que, en la población en general, hay un $16 \%$ de personas que terminan una carrera terciaria o superior, mientras que en el caso de la población con discapacidad visual, este porcentaje desciende a tan sólo un $10 \%$.

Por los datos anteriormente mencionados, y por la información aportada por las Organizaciones No Gubernamentales que trabajan con la discapacidad visual, se estima que existen en la provincia de Córdoba al menos $4000^{\mathrm{iii}}$ personas en esta franja etaria (algunos estudiando, otros no). Esa cifra se deduce teniendo en cuenta que el total de la población (INDEC 2003) asciende a 3.000 .000 de personas. El $8 \%$ de la población total posee discapacidad visual, que equivale a 240.000 personas. Se calcula que el $5 \%$ de esta última cifra -esto es 12.000 personas- está integrado en una institución educativa. Por ello, se deduce que al menos 4000 aspiran o están en la universidad o en una carrera terciaria.

El alto nivel de deserción registrado en la educación media y superior por parte de estudiantes con discapacidad visual tanto en Argentina como en Uruguay es la razón que motivó la elaboración de un diagnóstico para identificar las barreras causantes de esta situación y, consecuentemente, el diseño de una intervención específica para atender esta problemática.

A partir de ese diagnóstico, y de la experiencia de una alumna ciega de la Escuela de Ciencias de la Información de la UNC, se identificó la escasez de materiales accesibles y de formación específica para el aprovechamiento de lo que aportan las tecnologías de la comunicación y de la información adaptadas para ciegos. 
Por otro lado, un concepto muy difundido en la actualidad es el de la "brecha digital", que en realidad no es una sola sino que puede hablarse de distintos tipos de brecha: brecha de acceso a las tecnologías, brecha de uso y brecha de uso significativo.

La primera, brecha de acceso, se refiere a la posibilidad de contar efectivamente con el equipamiento necesario. En el caso de los estudiantes con discapacidad visual, además del hardware estándar, requieren de un soft específico lector de pantalla. La segunda brecha se refiere a las competencias para usar las TIC. De nada sirve producir materiales en soportes digitales si los usuarios carecen de las habilidades y destrezas necesarias para usarlos. La tercera brecha es la de uso significativo: se cuenta con el equipamiento, se sabe usar las funciones principales, pero se carece de las competencias para aprovechar todas las potencialidades de estas herramientas.

Teniendo en cuenta la situación anterior y los conceptos expuestos, se plantearon los cuatro aspectos sobre los cuales se trabaja: producción de textos accesibles, acceso público a las Tiflo-TIC, capacitación informática y empoderamiento.

\section{Metodología}

Como ya se mencionó, inicialmente se conformaron tres centros MATE en Córdoba, en Buenos Aires y en Montevideo-Uruguay, siguiendo procesos diferentes y con distintas fuentes de financiamiento, pero trabajando siempre de forma conjunta en la formulación de los proyectos y el diseño de las estrategias de trabajo.

En los centros de Buenos Aires y Montevideo se labora de manera asociada con la Facultad de Ciencias Sociales de la UBA y la Universidad de la República del Uruguay, respectivamente.

En el caso de Córdoba, la creación del centro fue posible a través del proyecto "Oportunidad para estudiantes con discapacidad visual: aprovechamiento de las TIC para promover la autonomía y el acceso igualitario a la educación", presentado en la convocatoria 2008 del Programa de Voluntariado Universitario de la Secretaría de Políticas Universitarias del Ministerio de Educación de la Nación. De esta manera, la Escuela de Ciencias de la Información incorporó las herramientas tecnológicas necesarias para iniciar con las actividades previstas. También, en la convocatoria 2009 de la Secretaría de Extensión Universitaria, se obtuvo una beca anual y un subsidio que permitió incorporar más hardware y contar con dos estudiantes dedicadas diariamente a coordinar las actividades del centro. 
La intención es aprovechar las posibilidades de conexión que ofrecen las herramientas tecnológicas para poner en común los recursos que se producen desde cada lugar, aunar esfuerzos, economizar los recursos disponibles atendiendo la mayor cantidad de necesidades posibles y fomentar la incorporación de otros centros, que trabajen en el desarrollo local de estos servicios, así como en la coordinación global de las acciones.

También se pretende que los/as estudiantes con discapacidad visual beneficiados en el marco de este proyecto conozcan a fondo las herramientas técnicas y estén en condiciones de aprovecharlas como medios para acceder a información y textos de lectura importantes para el desarrollo de su carrera, adquirir autonomía en el estudio y mejorar su desempeño académico y profesional. Además, se apunta a que ellos mismos sean protagonistas del proyecto y participen activamente como multiplicadores, acercando la información y la capacitación recibidas a otros jóvenes con discapacidad visual.

Al fortalecer y consolidar el trabajo de la Red MATE, se apunta a enfrentar el problema usando la tecnología no como un fin sino como un medio para la coordinación, la puesta en común y la optimización de los recursos y experiencias. Los niveles de participación y la calidad de la misma en ámbitos de educación superior amplían significativamente las oportunidades de inclusión social y de una mejor calidad de vida presente y futura.

Resumiendo, se propone utilizar las herramientas tecnológicas como puente para: la igualdad de oportunidades en el ingreso a la universidad; lograr un mejor desempeño académico, lo que a su vez redundará en un mejor desempeño profesional y mayores oportunidades de acceso a un empleo calificado. En síntesis, una participación igualitaria en el campo estudiantil, laboral y profesional.

\section{Aplicación}

\subsection{Situación contextual}

Actualmente, el desarrollo de las herramientas tecnológicas adaptadas para personas ciegas produce importantes cambios, tanto en la producción de materiales como en la posibilidad de acceso independiente a una amplia cantidad de información. Sin embargo, aún son pocas las personas que acceden a estas tecnologías, principalmente por el costo que implican, son escasas las opciones de entrenamiento y capacitación orientadas al aprovechamiento de las Tecnologías de Información y Comunicación adaptadas para ciegos como medios para el acceso igualitario a la educación; las instituciones educativas -incluidas las universidadesno ofrecen, en general, acceso a esas tecnologías a quienes no puedan hacerlo por sí mismas. 
Cabe destacar también que los instrumentos de recolección de información vigentes en la UNC -como la ficha de matriculación- no poseen indicadores que permitan identificar la cantidad de estudiantes con discapacidad visual que transitan por sus unidades académicas. Esto, sin dudas, es un inconveniente a la hora de plantear una intervención específica.

Asimismo, la experiencia de una alumna ciega de la licenciatura en Comunicación Social, miembro del equipo de gestión del proyecto, sirvió de ejemplo para reconocer la inexistencia de políticas universitarias tendientes a garantizar condiciones equitativas de acceso a todos los estudiantes. En la mayoría de las bibliotecas universitarias no se dispone de libros ni apuntes en soportes electrónicos accesibles, ni equipos informáticos con lectores o magnificadores de pantallas para el uso de este segmento de la población. Esto genera la necesidad de que cada estudiante con discapacidad visual deba procurarse el material por cuenta propia y en forma aislada, lo que hace mucho más problemático acceder a la lectura y, además, que el esfuerzo individual no sea aprovechado por todos.

Esta cuestión es la que de manera reiterada refieren los estudiantes ciegos: la existencia de esfuerzos aislados que intentan resolver los inconvenientes en el acceso a la lectura. Pero este trabajo fragmentado y desarticulado no permite abordar el problema en profundidad, sino que apunta a atenderlo parcialmente ante su eventual aparición. De esta manera, las posibilidades de permanecer y avanzar con el cursado de las materias de los estudiantes con discapacidad visual se encuentran supeditadas a la acción voluntaria y solidaria de terceros que se ocupen de leer o convertir en soportes accesibles los materiales impresos. A su vez, la falta de coordinación entre las instituciones educativas -incluidas las universidades y las instituciones que atienden a personas ciegas- genera que los esfuerzos se realicen de forma aislada y no se aprovechen todos los recursos existentes.

En este contexto, se propuso la Red MATE, cuyo pilar fundamental es el trabajo en red, coordinando entre diversos actores (instituciones de ciegos, centros educativos, organismos estatales, estudiantes con y sin discapacidad, etc.).

\subsection{Actividades}

De acuerdo con los ejes planteados, en dos años de trabajo se desarrollaron las siguientes acciones:

- Producción de materiales:

Se digitalizaron apuntes y libros para 108 materias de 12 carreras terciarias y universitarias de grado y posgrado, además de educación media para adultos. Los beneficiarios directos fueron 31 estudiantes de distintos niveles. 
Pero también los textos digitalizados se subieron a la documentoteca de la red MATE, por lo que también quedaron a disposición de los otros dos centros y de los más de 4.000 usuarios de todo el mundo que acceden a esa plataforma y a las 140 instituciones que brindan servicios para personas con discapacidad visual.

Trabajaron en la corrección de los materiales unas 150 personas entre estudiantes, egresados, docentes y no docentes. Hay que destacar que el voluntariado, además de cumplir tareas específicas como la corrección, ha sido una fuente de visibilización de la problemática, de movilización social y de diseminación de las ideas de la red. Eso ha sido muy positivo porque ha permitido reflexionar en conjunto sobre la inclusión, sobre qué significa tener condiciones equitativas, qué aportes puede hacer la red MATE y qué puede hacer cada integrante de la universidad, o mejor dicho de la sociedad.

Entre las actividades, se elaboró un Pequeño Manual para el Corrector, un instructivo que contiene cuestiones técnicas y recomendaciones para hacer más eficaz y homogénea la corrección. El material fue puesto a discusión con los otros dos centros, que hicieron aportes en función de sus experiencias, y fue adoptado para la corrección en toda la red.

- Acceso público a las Tiflo-TIC:

Para la misma época en que se creó el espacio de producción de materiales, se puso a disposición en la biblioteca de la ECI una PC con lector de pantalla Jaws para que los usuarios pudieran utilizar este espacio y consultar bibliografía y diferentes servicios accesibles a través de Internet.

También se invirtió en una computadora portátil con el software lector de pantalla, para que los estudiantes puedan rendir sus exámenes de forma escrita, al igual que el resto del alumnado. De esta manera, se avanzó en dar acceso a tecnología, no sólo en la biblioteca para uso particular de los estudiantes ciegos, sino también en la equiparación de las condiciones académicas de los mismos, en clases prácticas y o teóricas, exámenes escritos, etc.

- Capacitación en el uso de las Tiflo-TIC:

Dos personas se formaron en Tifo-TIC con la finalidad de transferir esos conocimientos a los estudiantes con discapacidad que lo solicitaran. Hasta fines de 2010 se habían realizado cuatro cursos de informática básica y avanzada.

Esta primera experiencia en formación resultó sumamente exitosa. En ningún caso se registró deserción, y la evaluación final de los alumnos respecto de sus aprendizajes fue muy positiva. En general, los alumnos valoraron la metodología de trabajo propuesta por los docentes partiendo de los saberes e inquietudes de cada uno. 
También, para algunos, significó una oportunidad para adquirir nuevas habilidades aplicadas al estudio, y a otros les permitió cumplimentar su formación académica y profesional.

- Empoderamiento:

Se organizó el primer encuentro de usuarios de la red MATE con la finalidad de vincular a estudiantes con discapacidad visual. Participaron 45 jóvenes entre 15 y 30 años de Córdoba, Buenos Aires y Uruguay, para compartir experiencias y desafíos, con el fin de generar estrategias que hagan posible el acceso al estudio en mejores condiciones.

Esta experiencia sirvió para movilizar a los destinatarios del proyecto, para involucrarse más activamente en el fortalecimiento de los objetivos propuestos. En este sentido, son los mismos estudiantes con discapacidad visual quienes tienen la mayor legitimidad para definir las dificultades, establecer líneas prioritarias de abordaje, y también proponer las mejores soluciones a los problemas identificados.

Uno de los resultados de este encuentro fue la constitución de un foro virtual de usuarios de la red MATE, integrado inicialmente por los asistentes al encuentro en Paysandú, pero abierto a todos los jóvenes estudiantes de la región que deseen subscribirse. A través de este medio se facilita la circulación de información relacionada con los intereses de sus miembros, se comparten experiencias, inquietudes, propuestas y se fomenta la participación en actividades presenciales de tipos educativas, culturales y recreativas.

El proyecto también desarrolló otras acciones complementarias pero tan necesarias para su funcionamiento como las anteriores. Ellas son:

- Contacto con instituciones externas al proyecto.

Desde noviembre de 2009 se inició una etapa de contacto interinstitucional, especialmente con la Oficina de Inclusión Educativa de Personas en situación de discapacidad de la Universidad Nacional de Córdoba, con la que se busca trabajar articuladamente a nivel local (derivación de casos que llegan a la Oficina de Inclusión, relación con los Secretarios de Asuntos Estudiantiles y propuestas de acción hacia el futuro).

También se lograron acuerdos con varias unidades académicas de la UNC para hacer más eficientes los procesos y optimizar los tiempos de corrección y entrega de materiales digitalizados. Éste ha sido un paso sumamente favorable para responsabilizar a la institución en el tratamiento de la temática puesto que no es el estudiante quien debe ocuparse de la accesibilidad de los apuntes sino que debe ser una responsabilidad institucional. 
Finalmente, se establecieron contactos con organismos públicos como el Ministerio de Educación de la Provincia (Programa de Integración Escolar y Diversidad, de la Subsecretaría de Promoción de la Igualdad y Calidad Educativa), y otros como el Instituto de Formación de Maestros Especiales Domingo Cabred, entre otros.

Finalmente, a través del Programa de Educación Solidaria, en agosto de 2010 se recibió una Mención Especial del Premio Presidencial Prácticas Educativas Solidarias en Educación Superior que otorga el Ministerio de Educación de la Nación. La actividad desarrollada por la Red MATE fue seleccionada junto a otras quince prácticas premiadas, entre 272 que se presentaron de todo el país.

- Difusión de la red MATE.

Se desarrollaron numerosas acciones de difusión, tanto para hacer conocer el servicio entre los potenciales usuarios como para convocar a voluntarios correctores y para sensibilizar a la población sobre la problemática de la accesibilidad académica para personas con discapacidad visual.

\section{Conclusiones}

Una de las principales fortalezas del proyecto es la alianza entre las universidades y las organizaciones de la sociedad civil, con especialización en la atención de las necesidades de las personas con discapacidad visual y el uso de la tecnología. El aval institucional de la Escuela de Ciencias de la Información de la UNC y la posibilidad de contar con un espacio en esa unidad académica ha sido de gran importancia para el desarrollo de la iniciativa. Al funcionar el centro MATE de Córdoba dentro del ámbito universitario se cuenta con recursos y logística de gran importancia. Los estudiantes solicitan y retiran los materiales en el mismo espacio universitario y el voluntariado para corrección de textos se desarrolla con mucha fuerza en este Centro, al trabajarse con estudiantes y docentes de las distintas unidades académicas.

A su vez Tiflonexos y la UNCU ha aportado al proyecto sus experiencias y sus conocimientos específicos, logrando así constituir una red sólida y eficiente en la que los diferentes participantes se complementan.

En dos años de funcionamiento de la Red Mate en su conjunto se han digitalizado más de mil textos (libros, apuntes, documentos, bibliografías) con un total de cerca de 150.000 páginas. Se 
atendieron pedidos de estudiantes de diversas carreras terciarias y universitarias y se digitalizaron textos de nivel medio y de educación secundaria para adultos tanto en Argentina como en Uruguay.

Si bien el impacto ya es importante, aún falta llegar a muchos jóvenes con discapacidad visual que estudian en diferentes carreras y no cuentan con un apoyo y atención específica. En la mayoría de las Universidades no se cuenta con información acerca de la cantidad de estudiantes que poseen una discapacidad visual. Muchos de ellos buscan resolver sus problemas de acceso a materiales de lectura mediante la ayuda de compañeros o familiares y desconocen aún las nuevas oportunidades que brinda el uso de las herramientas tecnológicas. Por ello hace falta ampliar el trabajo de difusión de estos servicios, tanto para llegar a nuevos estudiantes con discapacidad como para instalar en las universidades y en otros centros educativos la conciencia de la necesidad de la creación de condiciones para el acceso igualitario a la educación de estos grupos.

Otra fortaleza del proyecto es el constante intercambio de experiencias y la búsqueda de sistematización de prácticas y procesos. En dos años se han realizado encuentros de los tres centros de la red y actividades de capacitación, talleres y clínicas con los voluntarios y usuarios de los tres centros. A través de éstas se ha generado una planificación conjunta, se han tomado las buenas experiencias de cada centro y se ha trabajado en la creación de distintos documentos que recogen las formas de funcionamiento de la red y que serán insumos de gran importancia para la conformación de nuevos nodos en otras universidades u organizaciones de la sociedad civil.

Consideramos que atender las dificultades en el acceso a los materiales de estudio en soportes accesibles para estudiantes con discapacidad visual y brindar igualdad de oportunidades en el acceso a la educación, son cuestiones posibles de ser abordadas desde un enfoque multidisciplinario, en forma transversal, pues se apunta a promover el entrenamiento y uso de la tecnología como medio para generar la igualdad de oportunidades en el acceso y continuidad en la educación.

Al fortalecer y consolidar el trabajo de la Red MATE, se apunta a enfrentar el problema usando la tecnología no como un fin sino como un medio para la coordinación, la puesta en común y la optimización de los recursos. De esta manera, se aspira a reducir la deserción y 
alcanzar un mejor nivel académico de los estudiantes con discapacidad visual. Esto en conjunto, permitirá acceder a empleos calificados, lo que repercutirá en mejores niveles de inclusión social de este colectivo.

\subsection{Recomendaciones}

1) Incluir la variable discapacidad en la ficha catastral de inscripción a la educación universitaria a nivel nacional. Sabemos que esta alternativa existe en la Universidad Nacional de Río Cuarto y que les permite contactar rápidamente a los alumnos que tienen alguna discapacidad para poder actuar de manera rápida y oportuna. Otro antecedente es el de la Universidad de la República, en Uruguay, que incluyó la variable discapacidad por primera vez en el censo de la población estudiantil universitaria del año 2007.

Conocer cuántos alumnos con discapacidad visual cursan en una institución de nivel superior permite gestionar a tiempo los mecanismos o recursos que permiten favorecer la igualdad de condiciones durante el trayecto académico.

2) Sumar a la red nuevos espacios que trabajen en la producción de textos digitales, de modo de llegar a nuevos usuarios, compartir los recursos que ya están disponibles y multiplicar la capacidad productiva.

3) Proponer la centralización de la digitalización en los centros ya constituidos y la descentralización de la corrección en las distintas unidades académicas. Así se aprovechan los recursos ya disponibles y se fortalece el trabajo de corrección que por el momento se sustenta contrabajo voluntario.

4) Crear un repositorio virtual único de materiales de estudio de todas las unidades académicas. Con un solo repositorio todo el material estará disponible para todos los estudiantes de la UNC, accesibles desde todas las bibliotecas. Consideramos que esta modalidad de trabajo permitirá hacer más efectivo el proceso aprovechando los recursos humanos y técnicos disponibles, optimizando los tiempos de trabajo y evitando la 
superposición de esfuerzos (por ejemplo, que dos o más unidades digitalicen el mismo material). Esta mecánica implica, de todas maneras, un trabajo articulado con las bibliotecas y las Secretarías de Asuntos Estudiantiles

5) Contar con al menos una terminal de consulta en todas las unidades académicas, gabinetes de informática y bibliotecas. Los espacios de acceso público a Tiflo-TIC se conforman colocando computadoras adaptadas con software lector de pantalla y magnificadores de caracteres, en espacios de acceso público, para que quien posee una discapacidad visual y no tiene equipos propios pueda acceder a las oportunidades que hoy brinda el uso de estas tecnologías. En todos los casos se debe brindar acceso a Internet y a las plataformas virtuales que contienen todos los materiales aportados por la red.

6) Conformar un programa de capacitación continua, ya que no basta con colocar la tecnología para que esta tenga efectos transformadores sino que resulta necesario trabajar en la formación de los y las estudiantes con discapacidad visual, para que dominen las herramientas y conozcan las oportunidades que hoy brindan en cuanto a autonomía y acceso a la información. Esa capacitación se puede realizar de manera presencial o a distancia.

7) Trabajar con los jóvenes ciegos aspirantes a ingresar a la UNC, que cursan el último año del secundario, verdadero cuello de botella en lo que a deserción se refiere. Se considera que el ámbito propicio para ello es el Programa de Articulación con el Nivel Medio que desarrolla la Secretaría de Asuntos Académicos.

8) Brindar información a los docentes universitarios sobre las oportunidades que ofrecen las Tiflo-TIC en cuanto a la autonomía de los estudiantes ciegos o con baja visión y la equiparación de condiciones para el cursado y los exámenes. La difusión colabora en el mayor conocimiento de la temática y por lo tanto se favorece el desarrollo de actividades académicas inclusivas.

La principal preocupación es avanzar en la sostenibilidad del proyecto, sobre todo del Centro MATE Córdoba: sólo será posible a través de su institucionalización, es decir, de la 
apropiación de la problemática por parte de las autoridades universitarias y/o de las autoridades nacionales en materia educativa.

Finalmente, este proyecto sólo busca ser el punto de partida para pensar políticas educativas inclusivas que equiparen las condiciones de acceso, permanencia y egreso de las personas con discapacidad visual que estudian o aspiran a estudiar en la UNC. El objetivo hasta aquí fue identificar algunas barreras académicas que dificultan el desempeño académico de un estudiante con discapacidad visual, reconocer el grado de responsabilidad que tiene la institución en esta problemática y proponer una manera de abordar la situación. El desafío hacia el futuro es transitar un camino mucho más largo y complejo, que permita sentar bases y avanzar en la formulación de verdaderas políticas universitarias con la participación de las autoridades pertinentes, poniendo el acento en la diversidad de la población estudiantil.

\section{Bibliografía}

INSTITUTO NACIONAL DE ESTADÍSTICAS Y CENSOS. (2003). Encuesta Nacional de Discapacidad.

LEY DE EDUCACION SUPERIOR № 24.521 y su modificatoria № 25.573 y LEY № 26.285 de excepción al copyright.

HALLÚ, R. (2008): "La universidad pública tiene jerarquía” en Diario La Nación. Disponible en: http://www.lanacion.com.ar/nota.asp?nota_id=1003155

MARTIN BARBERO, J. (2009): "Es un escándalo que lo único que circule por América latina sean telenovelas" en Hoy la universidad. Disponible en: http://www.hoylauniversidad.unc.edu.ar/2009/diciembre/

MINISTERIO DE EDUCACIÓN, CIENCIA Y TECNOLOGÍA DE LA NACIÓN, SECRETARÍA DE POLÍTICAS UNIVERSITARIAS (2005): "La Integración de las Personas con Discapacidad en la Educación Superior en la República Argentina". Buenos Aires.

MINUJIN, A.; KESSLER, G. (2004): "La nueva pobreza en la Argentina. Empobrecimiento y educación- Las dos caras de la crisis educativa" en Apunte de la Cátedra de Teoría de la Educación eje II: Escenarios, políticas y actores en el campo educativo. Universidad Nacional de Córdoba. Escuela de Trabajo Social. 
Año 1 (2010) | Artículo no 11 | Págs. 214 - 228

ISSN: 2172 - 3168

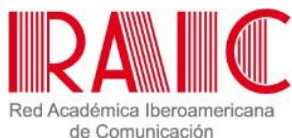

MORENO VALDÉS, M. T. (2006): “Integración/Inclusión de las personas con discapacidad en la Educación Superior". En: Informe sobre la Educación Superior en América Latina y el Caribe 2000-2005. La Metamorfosis de la Educación Superior. IESLAC - UNESCO. Ed. Metropolis. Caracas.

PUIGRÓS, A. (1996): "Educación neoliberal y quiebre educativo" en Nueva Sociedad. Nro. $146 . \quad$ Noviembre-diciembre $1996 . \quad$ Disponible en: http://www.nuso.org/upload/articulos/2549_1.pdf

SCOTTO, C. (2010): "Autonomía: El poder que nos obliga”. 90 aniversario. Página web de la Universidad Nacional de Córdoba. Disponible en: http://www.unc.edu.ar/reforma/analisis/participacion-comprometida-eindependiente/autonomia-el-poder-que-nos-obliga-por-carolina

\section{Forma de citar este artículo en bibliografías}

LOYOLA, M.I. y VIADA, M. (2010): "Nuevas tecnologías y accesibilidad académica para estudiantes con discapacidad visual”, en Revista PANGEA, 1, páginas 214 - 228. Red Académica Iberoamericana de Comunicación. Recuperado el _ de __ de 2 _ de: http://revistaraic.files.wordpress.com/2012/01/01-01-111.pdf

\footnotetext{
i Forman parte de este proyecto por la Escuela de Ciencias de la Información de la UNC: Doctoranda María Inés Loyola, Dra. Mónica Viada, Al. Jimena López, Al. Pamela Ussei, Dra. Mirta Echevarría, Lic. Judith Gerbaldo, Lic. Fabiana Castagno. ii El proyecto cuenta con distintas fuentes de financiamiento. En el nodo Córdoba, se financió con un Proyecto de Voluntariado de la Secretaría de Políticas Universitarias del Ministerio de Educación de la Nación y con una beca de la Secretaría de Extensión Universitaria de la Universidad Nacional de Córdoba. También en conjunto, la Red MATE accedió a financiamiento del Fondo de Cooperación Local de la Embajada de Finlandia.

iii - Población de la provincia de Córdoba (INDEC 2003): 3.000.000 de personas. El 8 \% de la población total posee discapacidad visual que, equivale a 240.000. Se calcula que el $5 \%$ de esta última población, 12.000 personas, está integrado en una institución educativa; por eso, podríamos estimar que, al menos 4000 aspiran o están en la universidad o en una carrera terciaria.
} 